\title{
Interactions Between COL5A1 Gene and Risk of the Anterior Cruciate Ligament Rupture
}

\author{
by \\ Ewelina Lulińska-Kuklik1, Masouda Rahim², Daria Domańska-Senderowska3, \\ Krzysztof Ficek4,5, Monika Michałowska-Sawczyn ${ }^{6}$, Waldemar Moska ${ }^{1}$, \\ Mariusz Kaczmarczyk1,7, Michat Brzeziański, Ewa Brzeziańska-Lasota3,

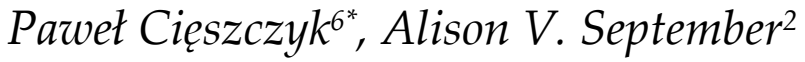

Collagen alpha-1(V) chain, encoded by the COL5A1 gene, plays a crucial role in abundant fibrillar collagens supporting many tissues in the body containing type I collagen and appears to regulate the association between heterotypic fibers composed of both type I and type $V$ collagen occurring among others in muscles, tendons and ligaments. Taking this fact into consideration we decided to examine the association between COL5A1 rs12722 and rs13946 polymorphisms, individually and as inferred haplotypes, with anterior cruciate ligament rupture risk (ACLR) in professional soccer players. A total of 134 male professional soccer players with surgically diagnosed primary anterior cruciate ligament ruptures and 211 apparently healthy male professional soccer players, who were without any self-reported history of ligament or tendon injury, were included in the study. Both the cases and the healthy controls were recruited from the same soccer teams, of a similar age category, and had a comparable level of exposure to anterior cruciate ligament injury. Genomic DNA was extracted from oral epithelial cells using GenElute Mammalian Genomic DNA MiniprepKit. All samples were genotyped for the rs12722 and rs13946 polymorphisms using a Rotor-Gene realtime polymerase chain reaction. Statistically significant differences in the genotype frequencies for the COL5A1 rs13946 polymorphisms in dominant modes of inheritance occurred $(p=0.039)$. Statistically significant differences were documented only in the dominant model under the representation tendency of the C-C haplotype in the ACLR group compared to controls $(p=0.038)$. Our results suggest that variation in the COL5A1 gene may be one of the nonmodifiable factors associated with the ACL injury in professional soccer players. The C-C rs12722-rs13946 haplotype provides a protective effect against the ACL tear.

Key words: COL5A1, ACLR, soccer players, injuries.

\section{Introduction}

The COL5A1 gene, localized on chromosome 9q34.3, encodes the alpha- $1(\mathrm{~V})$ chain of type $\mathrm{V}$ collagen. This minor fibrillar collagen plays acrucial role in the regulation of the size and

configuration of other abundant fibrillar collagens supporting many tissues in the body, such as tendons, ligaments, and muscles (Birk at al., 1990).

It has been shown that mutation in the

1 - Faculty of Tourism and Recreation, Gdansk University of Physical Education and Sport, Gdansk, Poland.

2 - Division of Exercise Science and Sports Medicine, Department of Human Biology, Faculty of Health Sciences, University of Cape Town, Cape Town, South Africa.

3 - Department of Molecular Bases of Medicine, 1st Chair of Internal Diseases, Medical University of Lodz, Poland.

4 - Faculty of Physiotherapy, The Jerzy Kukuczka Academy of Physical Education in Katowice, Katowice, Poland.

5 - Galen-Orthopaedics, Bierun, Poland.

6 - Faculty of Physical Education, Gdansk University of Physical Education and Sport, Gdansk, Poland.

7 - Pomeranian Medical University, Department of Clinical and Molecular Biochemistry, Szczecin, Poland. 
COL5A1 gene results in a $50 \%$ reduction of type $\mathrm{V}$ collagen and leads to poorly organized fibrils, decreased tensile strength, and reduced stiffness of connective tissue (Wenstrup et al., 2006). In addition, mutations within COL5A1 were implicated in Ehlers Danlos syndrome (Malfait et al., 2010; Myllyharju and Kivirikko, 2001), a condition characterized by joint hypermobility. The BstUI RFLP (rs12722) is a common C to T single nucleotide polymorphism (SNP) within the COL5A1 $3^{\prime}$ untranslated region which may alter COL5A1 messenger RNA (mRNA) stability (Laguette et al., 2011). Previous studies identified the BstUI restriction fragment length polymorphism (RFLP) to be associated with chronic Achilles tendinopathy (Mokone et al., 2006; September et al., 2009), anterior cruciate ligament ruptures (O'Connell et al., 2015; Posthumus et al., 2009; Petr et al., 2014), carpal tunnel syndrome (Burger et al., 2015) and properties of the knee ligament (Kubo et al., 2013). In addition, this SNP was also associated with joint flexibility (Brown et al., 2011), joint range of motion (ROM) (Collins et al., 2009) and endurance running performance (Brown et al., 2011; Collins and Posthumus, 2011). It was hypothesized that individuals with the rs12722 TT genotype have increased type $\mathrm{V}$ collagen production and thus favorably altered mechanical properties of tendons, which potentially enhances endurance running ability (Collins et al., 2011; Posthumus et al., 2011). Moreover, individuals with the COL5A1 $\mathrm{T}$ functional allele are reported to have an increased mRNA stability in vitro (Laguette et al., 2011). In these investigations, the TT genotype has been prominent with phenotypes of stiffer tendon and ligamentous mechanical properties (Laguette et al., 2011).

Mokone et al. (2006) reported a significant difference in the allele frequencies of the COL5A1 rs13946 DpnII RFLP between the Achilles tendon pathology group and control groups). However, research investigating a group of recreational skiers showed no significant differences in rs13946 (C/T) genotype frequency distributions between the ACL rupture and control groups (Posthumus et al., 2009; Stępień-Słodkowska et al., 2015).

The aim of this study was to investigate the rs12722 and rs13946 polymorphisms in the COL5A1 gene, individually and as haplotypes, with the risk of ACL ruptures in soccer players. We postulated that the COL5A1 rs12722 and rs13946 polymorphisms would be individually associated with ACL rupture risk and that an inferred haplotype of the two polymorphisms may provide more information into the potential role of COL5A1 in predisposing professional soccer players to higher risk of ACL rupture (O'Connell et al., 2015).

\section{Methods}

This study was approved by the Gdansk Medical University Ethics Committee, Poland (number KB 8/16) and written informed consent was obtained from each participant according to the declaration of Helsinki. A total of 134 professional male soccer players (age $=23.4 \pm 3.1$ years), with surgically diagnosed primary ACL ruptures who qualified for ligament reconstruction, were recruited for this study. All players had non-contact ACL ruptures. For the obvious reason that the soccer teams were homogenous in term of gender, we recruited only male subjects. All participants were soccer players playing in the Polish 1st division professional soccer league, with overall training time of 14-18 $\mathrm{h}$ per week (7-9 training sessions a week, $2 \mathrm{~h}$ each). Subjects were treated in the Galen Orthopaedics Clinic in Poland.

The control group consisted of 211 apparently healthy, male professional soccer players (age $=25.3 \pm 3.4$ years), without any selfreported history of ligament or tendon injury. Both the ACL rupture group and the healthy controls were from the same soccer teams, of the same ethnicity (all self-reported Polish, EastEuropeans for $\geq 3$ generations), and had a comparable level of exposure to risk of ACL injury (same volume and intensity of training as well as hours of match play).

We followed the STREGA recommendations for genotype-phenotype association studies (Little et al., 2009). An oral epithelium was collected from each participant using the DNA Swab (Copan, USA). Genomic DNA was extracted from the oral epithelial cells using a Gen Elute Mammalian Genomic DNA Mini prep Kit (Sigma, Germany) according to manufacturer's recommendations. Allelic discrimination of the COL5A1 rs12722 and rs13946 polymorphic sites (BstUI RFLP C/T and DpnII 
RFLP C/T, respectively) was performed using catalogued TaqMan ${ }^{\circledR}$ SNP Genotyping Assays (Applied Biosystems, USA). All samples were genotyped on a Rotor-Genereal-time polymerase chain reaction (PCR) instrument (Corbett, Australia) with the following cycling conditions: an initial hold step at $95^{\circ} \mathrm{C}$ for $5 \mathrm{~min}$, followed by 45 cycles of denaturation at $94^{\circ} \mathrm{C}$ for $15 \mathrm{~s}$ and anneal/extend at $60^{\circ} \mathrm{C}$ for $1 \mathrm{~min} .47$ positive (known genotypes) and negative controls (no DNA) were included on each PCR plate as quality control measures. Genotyping results were called by two independent, experienced investigators who were blinded to the participants' data.

\section{Statistical Analysis}

The programming language and environment $\mathrm{R}$ (http://www.r-project.org) was used for all statistical analyses. Genotype and allele frequencies were compared between the cases and controls using $\chi^{2}$ or Fisher exact tests. Allelic-based odds ratios (OR) with 95\% confidence intervals $(95 \% \mathrm{CI})$ were calculated using logistic regression analysis. The genotypes between cases and controls were compared in three ways: first, in a general test of association in the 2-by-3 table of phenotype-by-genotype, then two different modes of inheritance of the minor allele were assumed: dominant, in which homozygotes and heterozygotes for the minor allele were pooled and compared to homozygotes for the major allele and recessive, in which homozygotes and heterozygotes for the major alleles were pooled and compared to homozygotes for the minor allele. HardyWeinberg equilibrium probabilities and linkage disequilibrium (LD) between the SNPs were also calculated. The haplostats package was used to infer haplotype frequencies and to test the association between inferred haplotypes and the risk of ACL rupture assuming three possible haplotype effects: additive, dominant and recessive. Hap.score is the statistical co ref or haplotypes reflecting the strength of association; the positive value of Hap.score indicates increased risk of ACL injury for a particular haplotype, while a negative value indicates reduced risk. For all tests, significance was set at $p<0.05$.

Table 1

COL5A1 rs12722 C/T and rs13946 C/T allelic and genotypic frequency distributions in the anterior cruciate ligament rupture group ( $A C L$ rupture group) in comparison with the control group.

\begin{tabular}{|c|c|c|c|c|}
\hline \multicolumn{2}{|c|}{ SNP } & $\operatorname{CON}(n=211)$ & ACL $(n=134)$ & $p$ \\
\hline \multirow{5}{*}{$\begin{array}{c}\text { COL5A1 } \\
\text { rs12722 }\end{array}$} & TT & $62(29 \%)$ & $45(34 \%)$ & 0.661 \\
\hline & CT & 107 (51\%) & $66(49 \%)$ & $\mathrm{pD}=0.411$ \\
\hline & CC & $42(20 \%)$ & $23(17 \%)$ & $\mathrm{p}_{\mathrm{R}}=0.526$ \\
\hline & C allele & $191(45 \%)$ & $112(42 \%)$ & 0.371 \\
\hline & HWE & 0.729 & 0.888 & \\
\hline \multirow{5}{*}{$\begin{array}{c}\text { COL5A1 } \\
\text { rs13946 }\end{array}$} & TT & $94(45 \%)$ & 75 (56\%) & 0.091 \\
\hline & CT & $102(48 \%)$ & 49 (37\%) & $\mathrm{pD}_{\mathrm{D}}=0.039$ \\
\hline & CC & $15(7 \%)$ & $10(7 \%)$ & $\mathrm{p}_{\mathrm{R}}=0.902$ \\
\hline & C allele & 132 (31\%) & $69(26 \%)$ & 0.119 \\
\hline & HWE & 0.071 & 0.610 & \\
\hline
\end{tabular}

$p_{D}$ and $p_{R}$ are two-sided Fisher's exact test probabilities for dominant (CC+CT vs TT) and recessive (CC+CT vs TT) modes of inheritance of the minor allele (rs12722 and rs13946), respectively.

HWE: hardy Weinberg exact tests of significance 
Table 2

Analysis of the frequency distributions of the COL5A1 rs12722-rs13946 haplotype in the study group and the control group for the three genetic models - dominant, recessive and additive.

\begin{tabular}{|c|c|c|c|c|c|}
\hline $\begin{array}{l}\text { (Haplotype } \\
\text { (rs12722- } \\
\text { rs13946 }\end{array}$ & $\begin{array}{l}\text { Control } \\
(\mathrm{n}=211)\end{array}$ & $\begin{array}{l}\text { Study } \\
(\mathrm{n}=134)\end{array}$ & $\begin{array}{c}\text { Dominant } \\
\text { model (global- } \\
\text { stat }=4.35, p= \\
0.226 \text { ) }\end{array}$ & $\begin{array}{c}\text { Recessive model } \\
\quad \text { (global-stat }= \\
4.72, p=0.193 \text { ) }\end{array}$ & $\begin{array}{c}\text { Additive model } \\
\text { (global-stat }= \\
2.75, p=0.253 \text { ) }\end{array}$ \\
\hline
\end{tabular}

$\begin{array}{lrrrrr}\text { T-T } & 55 \% & 58 \% & 0.63, p=0.525 & 0.82, p=0.411 & 0.90, p=0.366 \\ \text { C-C } & 31 \% & 26 \% & -2.06, p=0.038 & 0.12, p=0.901 & -1.60, p=0.107 \\ \text { C-T } & 14 \% & 1 \% & 0.32, p=744 & 1.90, p=0.057 & 0.76, p=0.442 \\ \text { global-stat - association of all haplotypes of the trait; hap.score - measure individual haplotype } \\ \text { association to the trait; Significant } p \text {-values are in bold ( } p<0.05) .\end{array}$

\section{Results}

At baseline, male professional soccer players with surgically diagnosed primary ACL ruptures and the control group did not differ significantly according to age and the level of exposure to ACL injury. The genotype and allele frequencies for the COL5A1 rs12722 and COL5A1 rs13946 are shown in Table 1 . The genotype distributions for both polymorphisms met HardyWeinberg expectations in both groups.

There were no significant differences in the genotype or allele frequency distribution for either COL5A1 (rs12722) or COL5A1 (rs13946) polymorphisms between the control group and the ACL rupture group using the 2-by-3 general test of association (Table 1). Likewise, there were no significant differences in the genotype frequencies for the COL5A1 rs12722 polymorphisms, when dominant and recessive modes of inheritance were assumed. However, significant differences in the genotype frequency distribution were noted for the COL5A1 rs13946 polymorphisms when a dominant mode of inheritance was tested $(p=0.039)$ (Table 1$)$.

Evaluation of the haplotype frequency distribution for COL5A1 rs12722-rs13946 showed significant differences between the control group and the study participants. There were three haplotypes, T-T, C-C and C-T with frequencies of $56 \%, 29 \%$ and $15 \%$, respectively. Specifically the C-C haplotype was found to be overrepresented in the control group compared to the ACLR group $(p=0.038)$ (Table 2$)$ when the dominant model was tested. 


\section{Discussion}

Recent genetic research has summarised the specific markers to be associated with increased risk for sports injuries (Collins et al., 2015; Rahim et al., 2016; September et al., 2016) and performance-related conditions (Cupeiro et al., 2010; Maffulli et al., 2013; Wang et al., 2013). Use of this genetic information can aid in the identification of the biological pathways underpinning injury risk and may, in future, support the development of tailored injury prevention programmes for athletes and the application of targeted therapeutic interventions (Cięszczyk et al., 2017).

In our study, we examined the association between both COL5A1 rs12722 C/T (BstUI RFLP) and the COL5A1 rs13946 C/T (DpnII) polymorphisms individually and as haplotypes with anterior cruciate ligament rupture risk in professional soccer players. Our findings identified no significant differences in the genotype (under general model of association) and allele frequency distributions of any of these two polymorphisms between the control group and the ACL rupture group. However, the carriers of the rs13946 C allele were underrepresented in the ACL injury group compared with controls. Considering the lack of association in the allele-based test, our results are consistent with those previously reported by Stępień-Słodkowska et al. (2015) in recreational skiers from Poland. Similarly, we identified the TT haplotype (COL5A1 rs12722 C/T-rs13946 C/T) to be the most common (56\%). The similarities in the frequency distributions between the two independent studies may be a result of both study groups originating from Poland. Further research in larger sample sizes and in independent populations is needed to investigate the role of the rs12722 and the rs13946 polymorphisms in ACL rupture risk.

Previously the rs12722 CC genotype was found to be associated with ACL tears in females in a Caucasian study group from South Africa (Posthumus et al., 2009). In addition, the rs12722 SNP was also associated with the development of bilateral quadriceps tendon rupture (Longo et al., 2010) and both the rs12722 and rs13946 SNPs were identified as genetic risk factors for tennis elbow (Altinisik et al., 2015)

In this study we found significant differences in the genotype frequencies for the COL5A1 rs13946 polymorphisms in dominant modes of inheritance, underrepresentation tendency of the C-T haplotype in the ACLR group compared to controls.

However, the authors do note that the sample size in both studies is a major limitation for such genetic association study. It is therefore recommended that the number of subjects be increased for a more comprehensive risk profiling analysis for ACLR susceptibility.

Several additional polymorphisms within COL5A1 have also been associated with exerciserelated phenotypes and are good candidates for further investigation. The MIR608 rs4919510 CC genotype was significantly over-represented in tendinopathy participants compared to asymptomatic controls (Laguette et al., 2011). Interestingly, the rs4919510 SNP (on chromosome 10q24) was associated with the functional microRNA (miRNA) binding site for Hsa-miR-608 within the COL5A1 3'-UTR, thereby affecting COL5A1 mRNA stability in vitro. Hsa-miRNA-608 binds to a functional polymorphic cis-acting element within the COL5A1 3'-UTR (Abrahams et al., 2013; Laguette et al., 2011). Kirk et al. (2016) indicated that the COL5A1 rs1536482 (A/G) and rs12722 $(\mathrm{C} / \mathrm{T})$ may potentially influence quadriceps muscle-tendon stiffness, but not lowlevel contractile properties.

\section{Conclusions}

Our results suggest that variation in the COL5A1 gene may be one of the non-modifiable factors associated with the ACL injury in professional soccer players. The C-C rs12722rs13946 haplotype provides a protective effect against the ACL tear.

\section{Practical Implications}

Although there are no immediate clinical implications, these findings help identify the main biological pathways contributing to injury susceptibility. In future, these genetic risk factors could be included in multifactorial risk models to assess one's ACL rupture susceptibility. Moreover, this information could support the development of tailored injury prevention programmes and/or targeted therapeutic interventions. 


\section{References}

Abrahams Y, Laguette MJ, Prince S, Collins M. Polymorphisms within the COL5A1 3'-UTR that alters mRNA structure and the MIR608 gene are associated with Achilles tendinopathy. Ann Hum Genet, 2013; 77(3): 204-214

Altinisik J, Meric G, Erduran M, Ates O, Ulusal AE, Akseki D. The BstUI and DpnII Variants of the COL5A1 Gene Are Associated With Tennis Elbow. Am J Sports Med, 2015; 43(7): 1784-1789

Birk DE, Fitch JM, Babiarz JP, Doane KJ, Linsenmayer TF. Collagen fibrillogenesis in vitro: interaction of types I and V collagen regulates fibril diameter. J Cell Sci, 1990; 95: 649-657

Brown J, Miller C-J, Posthumus M, Schwellnus MP, Collins M. The COL5A1 gene, ultra-marathon running performance and range of motion. Int. J. Sports Physiol, Perform, 2011; 6(4): 485-496

Brown JC, Miller C-J, Schwellnus MP, Collins M. Range of motion measurements diverge with increasing age for COL5A1 genotypes. Scand. J. Med. Sci. Sports, 2011; 21(6): e266-72

Burger $\mathrm{M}$, de Wet $\mathrm{H}$, Collins $\mathrm{M}$. The COL5A1 gene is associated with increased risk of carpal tunnel syndrome. J Clin Rheumatol, 2015; 34(4): 767-774

Cięszczyk P, Willard K, Gronek P, Żmijewski P, Trybek G, Gronek J, Weber-Rajek M, Stasny P, Petr M, Lulińska-Kuklik E, Ficek K, Kemeryte-Riaubiene E, Maculewicz E, September S. Are genes encoding proteoglycans really associated with the risk of anterior cruciate ligament rupture? Biol Sport, 2017; 34:97-103

Collins M. Interactions between collagen gene variants and risk of anterior cruciate ligament rupture. Eur J Sport Sci, 2015; 15 (4): 341-350

Collins M, Mokone GG, September AV, van der Merwe L, Schwellnus MP. The COL5A1 genotype is associated with range of motion measurements. Scand J MedSci Sports, 2009; 19(6): 803-810

Collins M, Posthumus M. Type V collagen genotype and exercise-related phenotype relationships: a novel hypothesis. Exerc Sport Sci Rev, 2011; 39(4): 191-198

Collins M, September AV, Posthumus M. Biological variation in musculoskeletal injuries: current knowledge, future research and practical implications. Br J Sports Med, 2015: 49(23): 1497-1503

Cupeiro R, Benito PJ, Maffulli N, Calderon FJ, Gonzalez-Lamuno D. MCT1 genetic polymorphism influence in high intensity circuit training: a pilot study. J Sci Med Sport, 2010; 13: 526-530

Kirk EA, Moore CW, Chater-Diehl EJ, Singh SM, Rice CL. Human COL5A1 polymorphisms and quadriceps muscle-tendon mechanical stiffness in vivo. Exp Physiol, 2016; 101(12): 1581-1592

Kubo K, Yata H, Tsunoda N. Effect of gene polymorphisms on the mechanical properties of human tendon structures. Springerplus, 2013; 25 (2): 343

Laguette MJ, Abrahams Y, Prince S, Collins M. Sequence variants within the 3'-UTR of the COL5A1 gene alters mRNA stability: implications for musculoskeletal soft tissue injuries. Matrix Biol, 2011; 30(5-6): 338-345

Little J, Higgins JP, Ioannidis JP, Moher D, Gagnon F, von Elm E, Khoury MJ, Cohen B, Davey-Smith G, Grimshaw J, Scheet P, Gwinn M, Williamson RE, Zou GY, Hutchings K, Johnson CY, Tait V, Wiens M, Golding J, van Duijn C, McLaughlin J, Paterson A, Wells G, Fortier I, Freedman M, Zecevic M, King R, Infante-Rivard C, Stewart A, Birkett N. STrengthening the REporting of Genetic Association studies (STREGA) - an extension of the STROBE statement. Eur J Clin Investig, 2009: 39(4): 247-266

Longo UG, Fazio V, Poeta ML, Rabitti C, Franceschi F, Maffulli N, Denaro V. Bilateral consecutive rupture of the quadriceps tendon in a man with BstUI polymorphism of the COL5A1 gene. Knee Surg Sports Traumatol Arthrosc, 2010; 18(4): 514-518

Maffulli N, Margiotti K, Longo UG, Loppini M, Fazio VM, Denaro V. The genetics of sports injuries and athletic performance. Muscles Ligaments Tendons J, 2013; 3: 173-189

Malfait F, Wenstrup RJ, De Paepe A. Clinical and genetic aspects of Ehlers-Danlos syndrome, classic type. Genet Med, 2010; 12(10): 597-605

Mokone GG, Schwellnus MP, Noakes TD, Collins M. The COL5A1 gene and Achilles tendon pathology. Scand J Med Sci Sport, 2006; 16(1): 19-26

Myllyharju J, Kivirikko KI. Collagens and collagen-related diseases. Ann Med, 2001; 33(1): 7-21 
O’Connell K, Knight H, Ficek K, Leońska-Duniec A, Maciejewska-Karlowska A, Sawczuk M, StępieńSłodkowska M, O'Cuinneagain D, van der Merwe W, Posthumus M, Cięszczyk P, Collins M. Interactions between collagen gene variants and risk of anterior cruciate ligament rupture. Eur J Sport Sci, 2015; 15(4): 341-350

Petr, M., Štastný, P., Pecha, O., Šteffl, M., Šeda, O., \& Kohlíková, E. (2014). PPARA intron polymorphism associated with power performance in 30-s anaerobic Wingate Test. PloS one, 9(9): 107171.

Posthumus M, Schwellnus MP, Collins M. The COL5A1 gene: a novel marker of endurance running performance. Med Sci Sports Exerc, 2011; 43(4): 584-589

Posthumus M, September AV, O'Cuinneagain D, van der Merwe W, Schwellnus MP, Collins M. The COL5A1 gene is associated with increased risk of anterior cruciate ligament ruptures in female participants. Am J Sports Med, 2009; 37(11): 2234-2240

Rahim M, Collins M, September AV. Genes and musculoskeletal soft-tissue injuries. In M. Posthumus \& M. Collins, eds. Medicine and Sport Science, Basel, Karger, 2016; 68-91

September AV, Cook J, Handley CJ, van der Merwe L, Schwellnus MP, Collins M. Variants within the COL5A1 gene are associated with Achilles tendinopathy in two populations. Br J Sports Med, 2009; 43(5): 357-365

September AV, Rahim M, Collins M. Towards an understanding of the genetics of tendinopathy. In P. W. Ackermann \& D. A. Hart, eds. Advances in Experimental Medicine and Biology. Advances in Experimental Medicine and Biology, Cham: Springer International Publishing, 109-116; 2016

Stępień-Słodkowska M, Ficek K, Kaczmarczyk M, Maciejewska-Karłowska A, Sawczuk M, Leońska-Duniec A, Stępiński M, Ziętek P, Król P, Chudecka M, Cięszczyk P. The Variants Within the COL5A1 Gene are Associated with Reduced Risk of Anterior Cruciate Ligament Injury in Skiers. J Hum Kinet, 2015; 7 (45): 103-111

Wang G, Padmanabhan S, Wolfarth B, Fuku N, Lucia A, Ahmetov II, Cieszczyk P, Collins M, Eynon N, Klissouras V, Williams A, Pitsiladis. Genomics of elite sporting performance: what little we know and necessary advances. Adv Genet, 2013; 84: 123-149

Wenstrup RJ, Florer JB, Davidson JM, Phillips CL, Pfeiffer BJ, Menezes DW, Chervoneva I, Birk DE. Murine model of the Ehlers-Danlos syndrome. col5a1 haploinsufficiency disrupts collagen fibril assembly at multiple stages. J BiolChem, 2006; 281(18): 12888-12895

\section{Corresponding author:}

\section{Paweł Cięszczyk}

Gdansk University of Physical Education and Sport

Kazimierza Gorskiego Street 1

80-336 Gdansk, Poland

E-mail: cieszczyk@poczta.onet.pl 